Regards sur l'économie allemande

Bulletin économique du CIRAC

$74 \mid 2005$

Varia

\title{
Bade-Wurtemberg : une compétitivité à taille humaine
}

Isabelle Bourgeois

\section{CpenEdition}

Édition électronique

URL : http://journals.openedition.org/rea/180

DOI : $10.4000 /$ rea. 180

ISBN : 978-2-8218-0844-7

ISSN : 1965-0787

\section{Éditeur}

CIRAC

\section{Édition imprimée}

Date de publication : 1 décembre 2005

Pagination : $17-28$

ISSN : 1156-8992

\section{Référence électronique}

Isabelle Bourgeois, «Bade-Wurtemberg : une compétitivité à taille humaine », Regards sur l'économie allemande [En ligne], 74 I décembre 2005, document 2, mis en ligne le 19 juin 2008, consulté le 19 avril 2019. URL : http://journals.openedition.org/rea/180 ; DOI : 10.4000/rea.180 


\section{Bade-Wurtemberg : une compétitivité à taille humaine}

\section{Isabelle Bourgeois}

"Si le Land de Bade-Wurtemberg était un Etat et une économie autonomes, il serait champion du monde ". Voilà comment cette région se présente dans une plaquette destinée aux investisseurs, rappelant qu'en valeur des exportations par habitants (plus de $10000 €$ ) elle surclasse le Japon et les USA. Mais la fierté n'est pas dénuée d'humour. La présentation se poursuit ainsi : "Mais nous n'oserions jamais le proclamer haut et fort ». Un clin d'œil à l'image d'une tranquille et riche province de 'fourmis' économes, travailleuses et inventives dont est doté ce Land industriel et high tech qui rivalise de compétitivité avec la Bavière voisine. Et qui se plaît à rappeler que, s'il n'est que la troisième économie de la République fédérale en termes de PIB, il n'en surclasse pas moins l'Autriche, la Suède ou le Danemark au sein de l'UE. Surtout, le Land est la région la plus innovante d'Europe, loin devant l'lle de France et la Bavière en ce qui concerne la part du PIB consacrée à la R\&D (près de $4 \%$ ) ou le nombre de brevets par habitants (130 contre 115 pour la Bavière).

$A$ «l'alliance du laptop et de la culotte de peau » qui résume la politique d'innovation bavaroise (voir REA 40/99), le Bade-Wurtemberg préfère une politique de compétitivité plus discrète, construite sur deux axes : la promotion de la culture industrieuse régionale grâce à un intense effort de formation professionnelle et de soutien aux activités de transfert de technologies et de savoirs, surtout dans les PME; l'aménagement d'un cadre favorable au développement des pôles de compétitivité qui ont émergé au fil du temps sur les piliers de l'industrie régionale (et allemande) : automobile, construction mécanique, TIC, biotechnologies et chimie. Mais l'internationalisation de ces branches qui faisait la force du Land risque aujourd'hui d'en fragiliser la compétitivité...

Réputé pour son attractivité touristique et gastronomique, le Bade-Wurtemberg (capitale: Stuttgart), qui s'étend sur la rive droite du Rhin, de l'Alsace à la Suisse et à la Bavière, figure parmi les Länder les plus prospères d'Allemagne. Son PIB (292 milliards $€$ en 2004) représente $15 \%$ du total allemand et le place juste après la Rhénanie du Nord-Westphalie et la Bavière. C'est non seulement un poids lourd industriel, mais le site industriel allemand par excellence, fief de ces biens d'investissement qui tirent traditionnellement la production manufacturière et les exportations de la République fédérale : la construction mécanique, l'automobile et l'électrotechnique. II faut y ajouter une intense activité de R\&D : un quart des brevets déposés outre-Rhin est à mettre à l'actif de ce Land qui se présente volontiers comme "le pays des inventeurs et des philosophes " (das Land der Tüftler und Denker), qui fut le berceau de l'automobile au XIXe siècle et où on se plaît à rappeler que l'automobile y a même été inventée deux fois : par Gottlieb Daimler à Mannheim et par Karl Benz à Cannstadt. On y recense aujourd'hui des groupes comme DaimlerChrysler, Bosch, Mahle, Stihl, Kärcher, Heidelberger Druckmaschinen AG, Dürr AG, Carl Zeiss $A G$, Südzucker ou encore Roche, Michelin et Alstom, sans oublier Porsche.

La production manufacturière y contribue au PIB à hauteur de 35,8\% (2004; Arbeitskreis VGR der Länder), voire de $37,7 \%$ (ministère de l'Economie du Land). C'est beaucoup plus qu'en moyenne nationale, où la part de l'industrie

Le site industriel allemand par excellence...

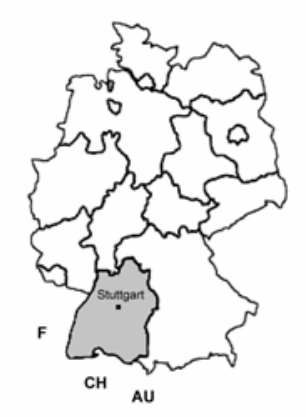

... mais une industrie de services 
est tombée à $28,8 \%$ selon les données publiées par le Land. C'est nettement plus aussi que dans cette Rhénanie du Nord-Westphalie qui fut longtemps le fief de l'industrie lourde mais qui, à l'issue d'une politique de restructuration originale (« du charbon au show biz puis au high tech », voir REA 62/03), a aujourd'hui largement tertiarisé son économie : la part des services y dépasse $71 \%$ (Arbeitskreis VGR der Länder). Elle n'est que de 63,2\% dans le BadeWurtemberg (61,5\% selon le ministère de l'Economie), ce qui, en termes relatifs, classe le Land au rang de l'économie la moins tertiarisée d'Allemagne. Mais ce qui semble un handicap recouvre en réalité le fait que les grands groupes industriels du Land ont intégré dans leurs structures leurs activités de services, et révèle également une très forte interdépendance 'intersectorielle' entre entreprises industrielles et prestataires de services, une structure dont le Land tire son avantage compétitif dans le jeu de la concurrence des sites allemands et européens. Car la moitié des activités tertiaires relève des services aux entreprises industrielles, ainsi que l'atteste la présence de sociétés comme SAP, ABB, Südleasing, Südfactoring ou Agilent Technologies Deutschland. Autrement dit: les deux tiers de l'activité du Land relèvent de cette industrie de services (voir REA 71/05) qui caractérise l'Allemagne ; la moitié seulement des activités tertiaires appartient au segment des services aux consommateurs.

Le poids respectif des Länder dans la République fédérale

\begin{tabular}{|c|c|c|c|c|c|c|c|c|c|c|}
\hline & $\begin{array}{c}\% \text { de la } \\
\text { superfic. }\end{array}$ & $\begin{array}{l}\% \text { de la } \\
\text { populat. }\end{array}$ & $\begin{array}{l}\% \text { des } \\
\text { actifs }\end{array}$ & $\begin{array}{c}\% \text { du } \\
\text { PIB }\end{array}$ & $\begin{array}{l}\% \text { de } \\
\text { l'ind. }\end{array}$ & $\begin{array}{c}\% \text { de } \\
\text { l'export }\end{array}$ & $\begin{array}{l}\text { PIB par } \\
\text { actif }^{\text {a }}\end{array}$ & $\begin{array}{l}\text { Crois- } \\
\text { sance }\end{array}$ & $\begin{array}{l}\text { Taux de } \\
\text { chômage }\end{array}$ & $\begin{array}{l}<25 \\
\text { ans }\end{array}$ \\
\hline Bade-Wurtemberg & 10,0 & 12,9 & 13,9 & 14,5 & 20,5 & 15,4 & 54614 & 1,6 & 6,2 & 6,0 \\
\hline Basse-Saxe & 13,3 & 9,7 & 9,0 & 8,5 & 8,8 & 6,6 & 49012 & 1,2 & 9,6 & 9,1 \\
\hline Bavière & 19,8 & 15,0 & 16,2 & 17,7 & 19,6 & 15,1 & 57442 & 1,9 & 6,9 & 7,3 \\
\hline Berlin & 0,2 & 4,1 & 4,0 & 3,5 & 1,9 & 1,4 & 46201 & 0,5 & 17,6 & 17,5 \\
\hline Brandebourg & 8,3 & 3,1 & 2,6 & 2,1 & 1,5 & 0,7 & 41073 & 0,9 & 18,7 & 17,4 \\
\hline Brême & 0,1 & 0,8 & 1,0 & 1,1 & 1,2 & 1,6 & 55278 & 1,0 & 13,3 & 11,0 \\
\hline Hambourg & 0,2 & 2,1 & 2,7 & 3,6 & 2,3 & 3,3 & 70827 & 1,4 & 9,7 & 8,1 \\
\hline Hesse & 5,9 & 7,4 & 7,8 & 9,1 & 8,0 & 4,9 & 61411 & 1,7 & 8,2 & 8,5 \\
\hline Mecklembourg & 6,5 & 2,1 & 1,9 & 1,4 & 0,7 & 0,3 & 39078 & 1,1 & 20,5 & 17,8 \\
\hline Rhénanie du N.- W. & 9,5 & 21,9 & 21,6 & 22,1 & 20,5 & 17,5 & 53815 & 1,4 & 10,2 & 9,1 \\
\hline Rhénanie-Palatinat & 5,6 & 4,9 & 4,6 & 4,4 & 5,2 & 3,9 & 50712 & 2,0 & 7,7 & 8,3 \\
\hline Sarre & 0,7 & 1,3 & 1,3 & 1,2 & 1,3 & 1,2 & 48652 & 1,9 & 9,2 & 9,9 \\
\hline Saxe & 5,2 & 5,3 & 5,0 & 3,7 & 3,0 & 2,0 & 38558 & 2,2 & 17,8 & 14,6 \\
\hline Saxe-Anhalt & 5,7 & 3,1 & 2,6 & 2,1 & 1,6 & 0,7 & 41867 & 1,2 & 20,3 & 17,2 \\
\hline Schleswig-Holstein & 4,4 & 3,4 & 3,2 & 3,1 & 2,3 & 1,7 & 51640 & 1,8 & 9,8 & 10,5 \\
\hline Thuringe & 4,5 & 2,9 & 2,7 & 1,9 & 1,8 & 0,7 & 37600 & 1,5 & 16,7 & 14,4 \\
\hline RFA & 100 & 100 & 100 & 100 & 100 & $<100^{b}$ & 52445 & 1,6 & 10,5 & 9,9 \\
\hline
\end{tabular}

Source : Arbeitskreis VGR der Länder pour les données relatives au PIB, à la croissance et aux taux de chômage (PIB aux prix de 1995) ; année 2004. Source : Destatis pour les données relatives à la superficie, la population, aux actifs, à la part dans l'industrie allemande et dans l'export ; elles se rapportent à 2002 (dernières données disponibles). a) en $€$. b) Une partie des exportations allemandes n'est pas imputable à un Land en particulier.

Le premier site exportateur d'Allemagne

Un gros tiers de l'export hors de la zone euro
Trois branches y réalisent les deux tiers du CA de la production manufacturière (62\% en 2004) et occupent $58 \%$ des actifs : la construction mécanique, l'automobile et l'électrotechnique. Elles exportent plus de la moitié de leur production. Ces branches réalisent $75 \%$ des exportations de l'ensemble de la production manufacturière bade-wurtembergeoise dont, toutes branches confondues, le taux d'exportation est de $45 \%$ aujourd'hui ; elle n'était encore que de $31,5 \%$ en 1995 , rappelle le ministère de l'Economie du Land. A elle seule, l'industrie automobile réalise plus d'un tiers $(35,5 \%$, soit 40 milliards $€)$ du total des exportations du Land, suivie de la construction mécanique (28 milliards $€$ ) et de l'électrotechnique (18 milliards $€$ ). Les performances du Land sont stratégiques pour la croissance allemande, comme le révèle la part de ces trois branches dans le total des exportations de la RFA par secteurs : respectivement 20,2 \% (automobile), 26,2 \% (construction mécanique) et 13,8 \% (électrotechnique). Le BadeWurtemberg est le premier site exportateur de l'Allemagne.

Près des deux tiers (58\%) des ventes s'effectuent au sein de l'UE ; c'est moins que la moyenne allemande (63,5\%). La France est le premier partenaire com- 
mercial européen du Land, avec $10,3 \%$ de l'export (idem pour le total allemand), suivie de l'Italie $(7,7 \%$; moyenne allemande : $7,1 \%)$, de la GrandeBretagne (7,1\% contre $8,3 \%)$ et de l'Autriche (5,3\% contre $5,4 \%)$. Le gros tiers restant des exportations se répartit entre les Etats-Unis et l'Asie. Hors de la zone Euro, le principal partenaire commercial du Bade-Wurtemberg sont les USA, avec $13,3 \%$ des ventes ; cette part nettement supérieure à la moyenne allemande $(8,8 \%)$ s'explique par la structure industrielle du Land où se concentre la production d'une part de ces biens d'investissement très demandés outre-Atlantique. Si quelque $12 \%$ des ventes sont réalisées sur la marché asiatique, la Chine occupe une part certes encore modeste (3\%), mais en très forte croissance.

Bade-Wurtemberg : industrie et structure des exportations en 2004

\begin{tabular}{|lccc|}
\hline Industrie & BW & RFA & Part dans RFA \\
Entreprises (nombre) & 8600 & 46600 & $18,5 \%$ \\
Salariés (en mio) & 1,212 & 6,019 & $20,1 \%$ \\
CA total (en mrd $€$ ) & 251 & 1421 & $17,7 \%$ \\
CA à l'export (en mrd $€$ ) & 113 & 560 & $20,2 \%$ \\
Taux d'exportation & $45,0 \%$ & $39,4 \%$ & - \\
\hline Exportations & BW & RFA & Part dans RFA \\
Total (en mrd $€$ ) & 114 & 731 & $15,6 \%$ \\
Automobile (en mrd $€$ ) & 27 & 135 & $20,2 \%$ \\
Construction mécanique (en mrd $€$ ) & 27 & 103 & $26,2 \%$ \\
Electrotechnique (en mrd $€$ ) & 17 & 123 & $13,8 \%$ \\
Chimie (en mrd $€$ ) & 11 & 95 & $11,3 \%$ \\
\hline
\end{tabular}

Source : Wirtschaftsministerium Baden-Württemberg, Jahreswirtschaftsbericht 2004/05

Ces fortes activités à l'export sont bien sûr le fait de groupes industriels mondialement réputés et fortement internationalisés. 83 des 500 plus grands groupes allemands sont ainsi implantés dans le 'pays' de Mercedes, Lidl, MTU, Voith, Würth ou encore Hugo Boss. Mais ils ne sont que la partie la plus visible d'un très dense tissu d'entreprises dominé par des structures petites et moyennes et qui constitue de véritables grappes d'activités. L'exemple le plus classique en est le secteur automobile avec ses réseaux intégrant l'ensemble de la chaîne de création de richesse, de la conception au segment des équipementiers. D'une manière plus générale, les entreprises-phares des branches les plus ouvertes à la compétition mondiale 'animent' ainsi littéralement ces réseaux via contrats et commandes, et surtout en diffusant vers l'amont leurs impératifs d'innovation et de compétitivité. Elles contribuent de la sorte à ce que se développe un environnement porteur, bénéfique à la croissance du site et à l'emploi.

Mais ces global players sont loin d'être les seuls. Car le Bade-Wurtemberg, que ses habitants surnomment le Ländle (le " bon petit 'pays' »), est la patrie du small business; il constitue le véritable socle des activités. Les PME représentent en effet l'écrasante majorité des entreprises, quelle que soit la branche. Une bonne moitié des établissements occupe au plus quatre salariés ; $2 \%$ seulement des entreprises en occupe plus de 50. La plupart des PME sont encore la propriété de la famille ou de la personnalité fondatrice. Nombre d'entre elles ont suivi une croissance continue au fil des générations. Certaines, désormais fortement internationalisées, figurent parmi les leaders mondiaux dans leur secteur. La liste comprend l'équipementier automobile Eberspächer $\mathrm{GmbH}$ \& Co. KG (Esslingen, CA : 1,47 milliard $€$ ), le spécialiste des tronçonneuses Stihl (Waiblingen, CA : 1,62 milliard $€$ ), transformé en société par actions en 1997 seulement, le fabricant de machines-outils et spécialiste de la découpe au laser Trumpf GmbH \& Co. KG (Ditzingen, CA : 1,4 milliard $€$ ), le spécialiste mondial de l'emballage aluminium ou plastique Hinterkopf $\mathrm{GmbH}$ (Eislingen, CA non communiqué), l'éditeur de jeux Ravensburger (Ravensburg, CA : 287 millions $€$,

Des groupes-phares mondialement réputés

Un dense réseau de leaders mondiaux de taille moyenne 
Un grand nombre de PME hautement compétitives

Un exemple de fonctionnement en partenariat : Hollister's MotorCycles

Des partenariats et une culture sources de compétitivité

Une forte intensité de R\&D société par actions depuis 1988), ou encore le fabricant de trains électriques miniatures Märklin GmbH (Göppingen, CA : 165 millions $€$ ).

Si ces entreprises de taille moyenne et supérieure sont mondialement réputées, la taille n'est pas le seul critère pour figurer parmi les hidden champions. On pourrait multiplier les exemples de ces petites et moyennes structures créées à l'origine autour d'un business modèle astucieux ou pour valoriser une invention. Elles sont nombreuses dans la région de Stuttgart, à forte densité industrielle, et dont les infrastructures de transport comme le haut niveau du capital humain sont particulièrement porteurs. On y trouve des références comme la société Leki Lenhart $\mathrm{GmbH}$ (Stuttgart), spécialiste mondial du bâton de ski high tech, issue en 1975 du plasturgiste Lenhart, le concepteur de lunettes de luxe Lunor AG, créée en 1991, ou encore le chocolatier Ritter-Sport GmbH \& Co. KG, aux mains de la famille fondatrice depuis 1920. Mais la capitale est loin d'avoir le monopole de ces champions, répartis également sur l'ensemble du territoire.

Une société est à cet égard un exemple révélateur, bien qu'elle constitue un cas particulier puisqu'elle œuvre dans une niche au sein du segment du luxe. C'est la Hollister's MotorCycles, développeur et constructeur de motos réalisées sur mesure. La manufacture artisanale, sise à Zimmern-Horgen dans la ForêtNoire (elle ne communique aucun chiffre), ne produit qu'une vingtaine de motos par an, mais toutes faites main et destinées à l'export (USA, à Monaco ou en Arabie Saoudite). Elle est représentative à sa manière de ce mode de fonctionnement en réseaux de partenariats constitué autour d'un business modèle original, en l'occurrence dans la niche de l'automobile individualisée de luxe. Parmi les partenaires de Hollister's figure ainsi une autre manufacture, créée en 1988, spécialiste aujourd'hui mondialement réputée des roadsters sur mesure : la Wiesmann $\mathrm{GmbH}$ \& Co. KG, elle-même partenaire de BMW pour la motorisation. Le réseau de Hollister's inclut par ailleurs un prestataire de services électroniques (IBS Internet Business Services; www.black-forest.de) ou le studio d'un photographe d'art (Peter Hillert; www.hillsalve.de) qui compte parmi ses clients Actea La Fenêtre confort ou Glaxo Smithcline Consumer Healthcare. S'y ajoutent l'hôtellerie de luxe (Interalpen Hotel Tyrol), de même que l'habillement sur mesure, avec les groupes belge Scabal et britannique Harper \& Fields.

La forte demande d'une offre individualisée dans le marché de masse par excellence qu'est l'automobile a incité l'équipementier Bosch à diversifier ses activités dans un créneau porteur, à la marge d'un segment du tuning en plein boom : celui des oldtimers. Au cours des dix dernières années, le nombre des véhicules de plus de 30 ans a en effet augmenté de $250 \%$ sur le seul marché allemand ; leurs propriétaires dépensent en moyenne quelque $1000 €$ pour leur entretien. Le groupe vient d'annoncer début novembre 2005 la création d'une nouvelle division opérationnelle : Bosch Automotive Tradition. Quatre pays sont visés, les fiefs de l'automobile de collection: Allemagne, France, Grande-Bretagne et Italie. On voit là comment, de proche en proche, se créent des synergies transsectorielles qui intègrent d'entrée de jeu l'espace européen ou mondial et dynamisent un territoire. Les entreprises sont insérées dans un environnement régional hautement compétitif, fondé sur une culture de la concurrence et de l'excellence qu'elles partagent toutes, quelle que soit leur taille. Cette culture n'est pas propre au seul secteur automobile, contrairement à ce pourrait laisser croire l'existence de champions dans le segment de l'équipement comme Behr GmbH \& Co. KG (Stuttgart ; CA : 3 milliards $€$ ), leader de la climatisation et du chauffage automobile. Ces champions se recrutent dans l'ensemble des activités, comme le montre l'exemple de la société $M+W$ Zander Holding AG (Stuttgart, CA : 2 milliards $€$ ) qui, en l'espace de 10 ans, a décuplé son CA et s'est hissée au rang des leaders dans le segment du facility management à partir de son cœur de métier : le microprocesseur.

La compétitivité de ces entreprises est liée à plusieurs facteurs. Le premier est la forte réactivité que permet leur taille petite ou moyenne, conjuguée à des cir- 
cuits décisionnels courts entre l'identification de la demande et la proposition de solutions au client. A cette forte proximité avec le marché s'ajoute un second facteur : la densité des activités industrielles et de services liés sur l'ensemble du territoire du Land. Autrement dit : les fournisseurs des entreprises citées et de tant d'autres sont eux aussi hautement réactifs et performants. C'est là le reflet du classique fonctionnement en réseau des PME allemandes. Enfin, troisième facteur : l'intensité de leurs activités de R\&D, particulièrement prononcée dans le Bade-Wurtemberg qui consacre quelque $4 \%$ de son PIB à la R\&D. Mais il s'agit là d'une moyenne. La société Trumpf, par exemple, consacre $8 \%$ de son CA à la R\&D - deux fois plus que la moyenne de sa branche. Elle s'est dès lors vu décerner en octobre le prix du Best of European Business (catégorie "Innovation »; entreprises au CA inférieur à 3 milliards $€$ ), créé dans sept pays européens (dont la France et la Grande-Bretagne) par le cabinet Roland Berger, le quotidien Financial Times et le périodique manager magazin. L'autre lauréat dans la catégorie innovation (CA supérieur à 3 milliards $€$ ) est le constructeur automobile bavarois BMW. Deux autres groupes du Bade-Wurtemberg se sont vu décerner le prix cette année, dans la catégorie « croissance exceptionnelle »: le constructeur automobile Porsche et le groupe pharmaceutique Altana AG (Constance, CA : 3 milliards $€$ ).

\section{Benchmarking régional des pôles de compétitivité allemands en termes de potentiel}

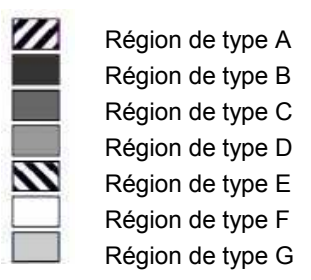

La carte ci-contre détaille le potentiel - non pas l'état actuel - de compétitivité des 439 circonscriptions administratives et agglomérations recensées en Allemagne, tel qu'il a été établi par Prognos. L'Institut a déterminé à cet effet sept types de profils ; ils se composent de 4 catégories d'indicateurs : démographie, marché de l'emploi, concurrence et innovation, prospérité et situation sociale.

La carte révèle que la quasi-totalité du Bade-Wurtemberg appartient au moins au type C. Ce classement s'explique par la forte compétition régionale au sein de la RFA. Si le Bade-Wurtemberg est hautement compétitif aujourd'hui, d'autres Länder se caractérisent par un dynamisme prononcé qui pourrait à l'avenir modifier sa position. Dans cette approche de benchmarking, seules l'agglomération de Stuttgart et ses environs, de même que le district du Lac de Constance ou la région Rhin-Neckar figurent parmi les clusters allemands (type A) les mieux armés pour l'avenir.

\begin{tabular}{|c|c|c|c|c|}
\hline Type & Démog. & Emploi & Concur./Innov. & Prospérit. \\
\hline A & ++ & ++ & ++ & ++ \\
\hline B & ++ & ++ & + & -- \\
\hline C & ++ & 0 & 0 & ++ \\
\hline D & + & 0 & - & + \\
\hline E & - & 0 & - & - \\
\hline F & - & - & -- & - \\
\hline G & -- & -- & -- & -- \\
\hline
\end{tabular}

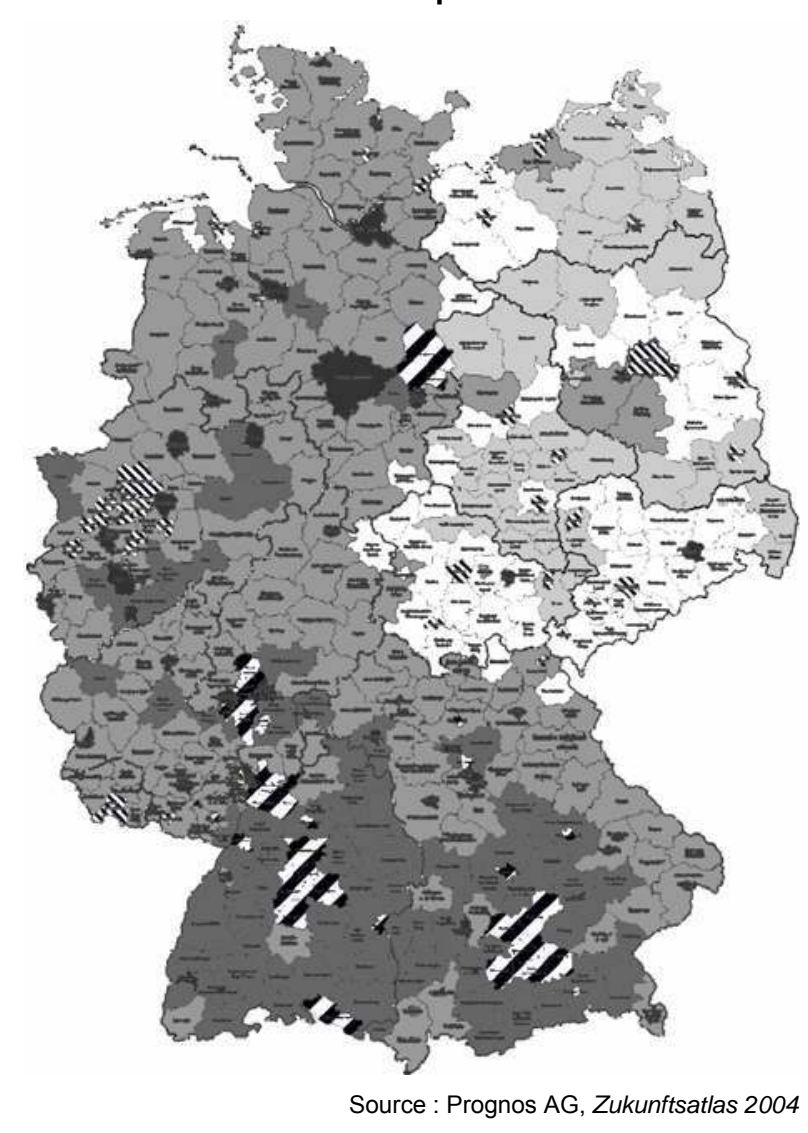

Le quatrième facteur est une caractéristique unique outre-Rhin, qui distingue le Bade-Wurtemberg des autres Länder, même de la Bavière voisine : une grande homogénéité territoriale en termes d'activités, de productivité ou d'innovation, c'est-à-dire l'absence de disparités régionales prononcées. Les activités sont certes concentrées dans les zones urbaines ; Stuttgart, Böblingen et Heidelberg constituent le trio de tête des agglomérations les plus compétitives. Mais les banlieues ou les zones à faible densité de population ne sont pas moins dynamiques. Près de la moitié des régions du Land sont hautement compétitives, ce
Homogénéité territoriale et partage interrégional du travail 
qui porte la part du Bade-Wurtemberg à $20 \%$ de l'Allemagne, estime l'institut Prognos. L'ensemble du Land constitue dès lors comme un pôle de compétitivité d'un seul tenant. Bien sûr, la structure des activités varie au sein du Land, délimitant son territoire en quatre principaux bassins qui suivent grosso modo les frontières des quatre districts administratifs (et des territoires des douze chambres de commerce et d'industrie). Or ces différences donnent lieu à une sorte de " partage interrégional du travail » (Arndt et al., 2005), à l'origine de la compétitivité du Bade-Wurtemberg, puisqu'il associe zones dédiées au savoir et aux services, donc à capital humain hautement compétitif, et zones dédiées à l'industrie et la R\&D, à forte intensité en capital.

4 districts administratifs...

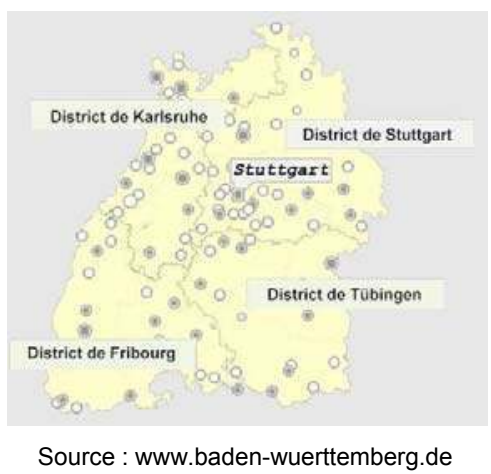

Le Bade-Wurtemberg $\left(35752 \mathrm{~km}^{2}\right.$, soit $5000 \mathrm{~km}^{2}$ de plus que la Belgique) est le seul Land allemand à avoir été créé par referendum. Il est issu en 1952 de la fusion de trois Länder créés après la guerre dans les zones d'occupation américaine (Nord-Württemberg-Baden) et française (Süd-Baden et Süd-Wurttemberg-Hohenzollern). Les districts administratifs actuels en suivent les anciennes frontières.

Depuis 1952, le parti CDU est majoritaire au sein du Land, composant le gouvernement tantôt seul, tantôt au sein d'une grande coalition avec le SPD (1966-72 et 1992-96), ou en s'alliant aux libéraux, ce qui est le cas actuellement. Ministre-président: Günther $H$. Oettinger (CDU).

Stuttgart :

$5,78 \%$ du PIB pour la R\&D

Automobile et construction mécanique tirent l'innovation
... et un réseau de $12 \mathrm{CCl}$

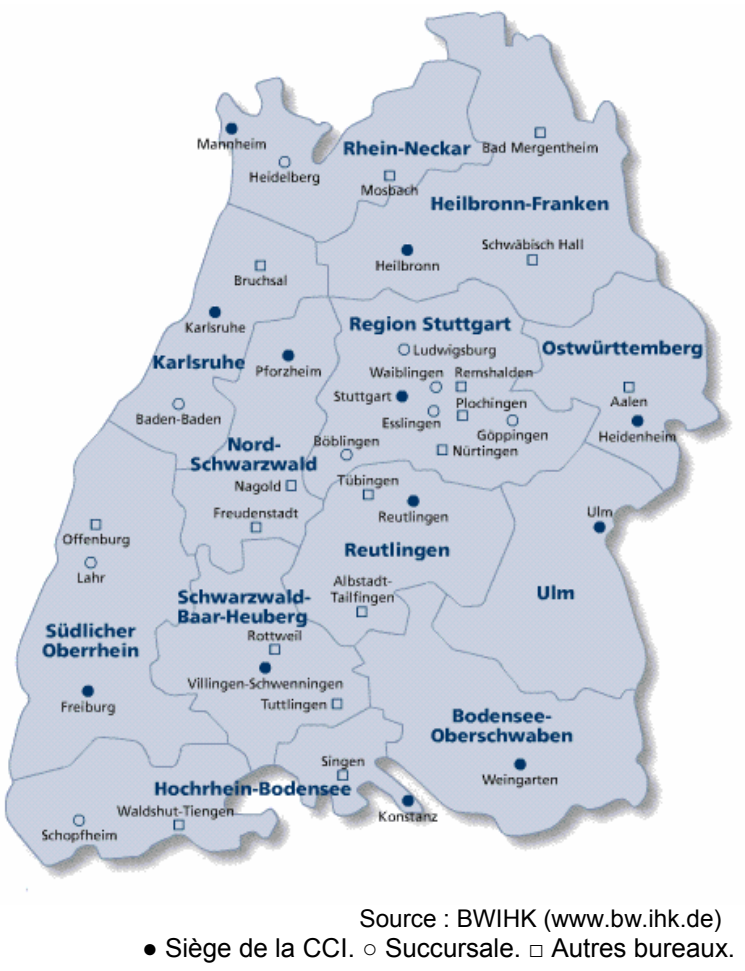

En matière d'industrie et de R\&D, une région domine de loin : celle de Stuttgart. La capitale du Land héberge l'une des six bourses régionales allemandes (voir $R E A 73 / 05$ ) et le siège de 22 grands groupes, ce qui la classe en $5^{\text {ème }}$ position après Hambourg, Munich, Düsseldorf et Essen, comme le rappelle une étude commanditée à Prognos par la banque publique du Land (L-Bank). Quatre branches y dominent : l'automobile, l'électrotechnique et l'informatique, l'édition et les médias, ainsi que l'assurance. Cette structure fait de la capitale la métropole la plus industrielle d'Europe : $40 \%$ de la création de valeur y est imputable à l'industrie (Werner et al., 2005) ; c'est 10 points de plus que les agglomérations de Milan et Turin, ou que la moyenne européenne. Elle lui confère aussi la seconde place parmi les métropoles les plus créatrices de richesse d'Allemagne, avec un PIB par habitant (en équivalents de pouvoir d'achat) de 31530 unités, après Munich (40500). Au sein de l'UE, elle occupe la $9^{\text {eme }}$ position (Munich, Londres et Copenhague constituant le trio de tête). Car à la différence des autres métropoles où dominent de loin les services, la structure majoritairement industrielle de Stuttgart a pour corollaire un énorme potentiel d'innovation. L'Office des statistiques du Land y a dénombré 876 brevets déposés par million d'habitants en 2002 ; c'est certes moins que Munich, mais la région de Stuttgart investit 5,78 \% de son PIB dans la R\&D - un record outre-Rhin, selon la CCI.

Cela s'explique par la forte présence de l'automobile et de la construction mécanique, les traditionnels moteurs de l'innovation incrémentale outre-Rhin (voir 
REA 71/05). A titre d'illustration : le "Prix Avenir » allemand 2005 (Deutscher Zukunftspreis) a été décerné le 11 novembre par le président de la République à une équipe pilotée par deux ingénieurs, employés respectivement par Siemens (Bavière) et Bosch (Stuttgart). Ils avaient développé le système d'injection piézoélectrique pour les moteurs diesel et essence. La présence de ces branches-phares entretient un environnement favorable à la création de start ups technologiques comme cette Foldcore $\mathrm{GmbH}$ (www.foldcore.de), créée en 2004 par deux ingénieurs sur la base d'un procédé du même nom développé par eux. Le procédé de pliage 'en sandwich' de matériaux fins, mais souples, comme le carton ou les tôles permet de produire à faibles coûts des matériaux légers, drainants, acoustiquement isolants, destinés au BTP, à l'automobile ou à l'aéronautique. Un de ses premiers clients est d'ailleurs Airbus. Or l'intense activité industrielle dope aussi le segment des services aux entreprises, comme le révèle le parcours de la Bernd Kußmaul GmbH (Weinstadt/ Großheppach), créée en 1997 par un technicien de la construction mécanique et diplômé en sciences de gestion (www.bernd-kussmaul-gmbh.de). Sa société, qui occupe aujourd'hui 15 salariés, s'est spécialisée dans le management de projet et le supply chain management; parmi ses premiers clients figuraient Audi Quattro $\mathrm{GmbH}$ et Adidas.

Au sein de la région de Stuttgart, un site s'est spécialisé dans le développement de logiciels et de hardware : l'agglomération de Böblingen/Sindelfingen au sudest, où sont installées des sociétés comme Hewlett Packard, Agilent Technologies, Philips Semiconductors, le second centre de recherche mondial d'IBM ou la Smart GmbH (DaimlerChrysler). Elle constitue un cluster certes particulier, mais intégré dans la structure des activités de la région. Ces groupes se sont associés avec les pouvoirs municipaux et régionaux dans une "Initiative de développement économique » au sein de laquelle ils mettent en réseau leur savoir-faire afin de le rendre accessible aux autres entreprises du cru, notamment aux PME. Dans ce cadre, le gouvernement du Land, les municipalités et la CCI locale ont lancé en 1995, avec le soutien de DaimlerChrysler, HP et IBM, un «Centre du logiciel » (www.softwarezentrum.de) rassemblant dans un réseau virtuel de coopération 80 PME du secteur, anciennes et nouvelles.

Si la région de Stuttgart est le leader incontestable parmi les zones dédiées à l'industrie et la R\&D, elle n'est pourtant pas la seule. A l'est, l'agglomération d'Ulm et, au sud, la région Bodensee-Oberschwaben font partie elles aussi de ces pôles industriels à fort dynamisme. Cette dernière région, qui partage le lac de Constance avec la Suisse et l'Autriche, héberge de grands groupes tels ZF Friedrichshafen, MTU, EADS Astrium ou EADS Dornier, mais aussi des entités moyennes comme VA Tech Escher Wiss $\mathrm{GmbH}$, Hawera Probst GmbH (outillage), sans oublier Ravensburger. La région, pourtant de structure à dominante agri- et sylvicole, compte parmi les plus dynamiques en termes d'innovation, avec 106 brevets pour 100000 habitants selon une étude de Prognos effectuée en 2002 (et citée par la CCl), ce qui la situe aujourd'hui au quatrième rang des régions d'Allemagne. Elle doit sa compétitivité à un recentrage collectif sur son cœur de compétences : l'automobile et la construction mécanique - les deux branches les plus en pointe à l'international.

L'agglomération d'Ulm, stratégiquement bien située sur l'axe Munich-Stuttgart, se caractérise quant à elle par le développement d'un cluster mettant en synergie la R\&D d'entreprise avec la recherche fondamentale et appliquée de l'université et des établissements extra-universitaires dans des domaines à première vue disparates, mais dont la coexistence reflète en réalité la diversité des enjeux liés aux technologies-clefs : médecine, chimie-biologie, physique, informatique ou mathématiques, sans oublier les technologies liées à l'énergie ni les sciences économiques et de gestion. Par ailleurs, tous les niveaux de qualification sont ainsi rassemblés - du technicien formé dans la Fachhochschule à l'ingénieur diplômé de l'Université d'Ulm -, ce qui contribue à une large diffusion de la culture de l'innovation, favorable à la réactivité des entreprises comme à
Böblingen/Sindelfingen : un cluster dédié au logiciel

Bodensee-Oberschwaben : recentrage sur le cœur de métier

Ulm : «Cité des sciences » et des technologies du futur 
Heidelberg, leader des services et des technologies du futur

Une tertiarisation en harmonie avec la recherche et l'industrie la promotion de la recherche fondamentale. On y observe une forte concentration de centres réputés comme l'Institut für Lasertechnologien in der Medizin und Meßtechnik (ILM, technologies du laser appliquées à la médecine et aux mesures et asservissement), I'Institut für Diabetestechnologie (technologies du diabète) ou le Zentrum für Sonnenenergie- und Wasserstoffforschung (ZSW, énergie solaire et recherche sur l'hydrogène). S'y ajoute le centre de recherche de DaimlerChrysler, spécialisé notamment dans les matériaux, la microélectronique ou l'énergie. Ces compétences sont réparties dans l'ensemble de l'agglomération, mais mises en réseau grâce à une politique de coopération lancée en 1986 et associant entreprises, pouvoirs locaux et dispositif de recherche dans une approche partagée de transfert des savoirs et compétences. Cette stratégie qui fait d'Ulm une «Cité des sciences » (Wissenschaftsstadt) avait pour objectif délibéré de permettre la reconversion d'une ancienne région industrielle vers un site compétitif dans l'économie du savoir. Dans le cadre de sa politique d'urbanisme et de développement économique, la municipalité a piloté et soutenu la création de trois Science Parks. "On ne peut pas forcer le succès; mais on peut optimiser les conditions-cadre », explique la ville (www.ulm.de); l'étude 2004 de Prognos sur la compétitivité des sites valide a posteriori cette approche. Elle se fonde aussi sur une coopération étroite avec l'agglomération voisine de Neu-Ulm, située sur la rive bavaroise du Danube, en matière de politique structurelle virtuelle (e-gouvernement). Cette politique interrégionale s'est vu décerner en novembre 2005 le Prix « Best-Practice-Award MEDIA@KommTransfer » décerné conjointement par le ministère fédéral de l'Economie et diverses entreprises dont SAP et T-Systems.

Dans la catégorie des régions dédiées aux services et aux savoirs, la ville de Heidelberg se profile comme leader en matière de biotechnologies, de TIC et de services financiers. Elle est au cœur de cette BioRegion Rhein-NeckarDreieck (triangle Mannheim, Ludwigshafen, Heidelberg au confluent du Neckar et du Rhin) qui est l'un des trois sites dédiés aux sciences du vivant lauréates du concours BioRegio lancé par le ministre fédéral de la Recherche en 1996 et destiné à rapprocher la recherche fondamentale du marché (voir REA 57/02). Dans ce cluster se concentrent en effet d'une part des structures réputées de recherche en biologie moléculaire comme le laboratoire européen EMBL créé en 1974, le centre de recherche ZMBH de l'Université Ruprecht-Karls, l'Institut Max-Planck de recherche médicale ou le centre de recherche allemand sur le cancer DKFZ, une fondation du Land de Bade-Wurtemberg qui est membre de la société Helmholtz et de la DFG. Ces structures, spécialisées notamment dans la biogénétique ou la bioinformatique, et qui sont toutes à Heidelberg, travaillent en réseau avec les pôles de technologie moléculaire, d'informatique et de mathématiques appliquées de Mannhein, ceux de microbiologie de Kaiserslautern ou ceux de biogénétique de Neustadt/Weinstraße (biotechnologies vertes). S'y ajoutent d'autre part des géants de la chimie/pharmacie installés dans les sites alentour (Roche Diagnostics, Merck, Abbott, Baxter, ou Aventis Pasteur) et un certain nombre d'entreprises innovantes à fort potentiel de croissance (Lion Biosciences, Leica Microsystems GmbH...). La plateforme technologique de Heidelberg (Life-Science-Park), sise sur le campus de l'université, est la première d'Allemagne, avec 45 entreprises et quelque 1000 salariés.

La ville de Heidelberg ( $80 \%$ des activités dans le tertiaire) est aussi un de ces pôles où se concentrent les services aux entreprises (l'éditeur de progiciels SAP, le courtier en assurances MLP AG ou les éditions scientifiques Springer). Or cette tertiarisation ne se fait pas au détriment de l'industrie, qui au contraire s'insère parfaitement dans ce schéma d'innovation, comme le révèlent les noms des entreprises qui ont leur siège dans la ville: Heidelberg Druckmaschinen AG (presses d'imprimerie, leader mondial), Heidelberger Zement AG (cimenterie), ABB AG, spécialiste de la robotique et des technologies de l'énergie, ou encore le fabricant de stylos Lamy $\mathrm{GmbH}$, une PME familiale au CA de 50 millions $€$. En termes de potentiel de compétitivité, la ville du philosophe 
Kant (www.heidelberg.de), pôle universitaire d'excellence, surclasse Stuttgart ; elle bénéficie d'une structure démographique particulièrement dynamique, avec une forte proportion d'actifs jeunes et hautement qualifiés.

Deux autres pôles urbains tertiaires se distinguent : Fribourg-en-Brisgau et Karlsruhe. La structure économique de ces villes universitaires et touristiques est dominée par la banque et l'assurance, le logiciel (autrement dit : des services aux entreprises), ainsi que les technologies liées à l'environnement. Fribourg est ainsi le premier pôle de compétence allemand dans le domaine de l'énergie solaire, ce qu'expliquent la présence de l'Institut Fraunhofer spécialisé en la matière (ISE), l'implantation d'entreprises comme Solarfabrik AG et Solarstrom AG ou d'institutions comme I'International Solar Energy Society (ISES), sans oublier les activités du principal salon européen du secteur, I'Intersolar. Parallèlement, la situation géographique de Fribourg (ouverture sur trois marchés nationaux) comme son dispositif de recherche universitaire et appliquée ont amené cette autre BioRegio allemande à s'associer avec les villes limitrophes (Strasbourg, Bâle et Karslruhe) dans un réseau de coopération transfrontalière : la Biovalley du Rhin supérieur, pôle biotechnologique d'envergure européenne.

La Technologiefarik de Karlsruhe (www.technologiefabrik-ka.de) est l'une des premières pépinières et plateformes technologiques créées en Allemagne, à l'initiative du Land qui lançait la, dès 1983, les axes d'une politique de développement structurel et de compétitivité globale cherchant avant tout à favoriser la mise en réseau des compétences. Avec deux objectifs : encourager une approche proactive de l'innovation dans les PME, et promouvoir la création de start ups technologiques grâce à une politique d'offre, ce qui distingue la politique du Bade-Wurtemberg de celle menée dans l'Alsace voisine, où la priorité est la sensibilisation à l'innovation (voir REA 54/01). Rappelons que l'existence d'une forte culture d'innovation est l'une des caractéristiques foncières du Bade-Wurtemberg - et ce, depuis le milieu du XIX siècle. La « Fabrique technologique » de Karlsruhe, installée dans l'ancienne manufacture de machines à coudre Singer à quelques minutes de l'université, est ainsi reliée à un plus vaste réseau régional d'incitation à la création d'entreprise, à la recherche fondamentale et appliquée, et au soutien au Mittelstand.

Et elle est membre de la fédération de ces plateformes (ADT) qui compte aujourd'hui 166 adhérents, dont 17 dans le Bade-Wurtemberg. Au fil du temps, quelque 220 entreprises sont nées dans l'incubateur de Karlsruhe qui accueille la $4^{\text {ème }}$ génération de créateurs. Sur ce total, huit start ups seulement ont abandonné, soit un taux de réussite de $96 \%$. Les compétences se concentrent actuellement sur les mesures et asservissement (fondamentales notamment dans le domaine des éco-industries, voir REA 57/02), les technologies au laser, ainsi que les TIC. Les partenaires institutionnels de la fabrique sont le Land, sa banque publique L-Bank et la $\mathrm{CCl}$, qui apportent financement, conseil et formation. S'y ajoutent les liens avec diverses fédérations professionnelles, des réseaux de créateurs d'entreprise ou de développeurs technologiques. La fabrique travaille, cela va sans dire, en coopération avec les établissements d'enseignement supérieur de Karlsruhe comme avec trois instituts du réseau de recherche appliquée Fraunhofer: I'ICT (technologies de la chimie), I'IITB (informatique) et l'ISI (innovations systèmes).

Le 'partage du travail' entre ces deux catégories de zones de compétitivité se nourrit d'une longue tradition manufacturière, ancrée dans un tissu très dense d'entreprises du Mittelstand particulièrement réactives à l'évolution du marché, et rompues dans l'art d'établir partenariats et réseaux (voir REA 64/03). «Les clusters sont souvent des unités géographiques de petite taille, nées spontanément », surtout dans l'industrie métallurgique et électrotechnique (Lichtblau et al., 2005). La part dominante réservée dans la structure des activités du Land à ces branches très internationalisées a assurément joué un rôle déterminant dans l'ouverture à l'international de l'ensemble des activités, de même qu'elle
Fribourg : ville solaire et membre de la BioValley

Karlsruhe : une « fabrique » des technologies...

... insérée dans des réseaux de coopération

Politique structurelle : amélioration du cadre réservé aux activités... 
... investissements dans le capital humain... entretient un climat favorable à l'innovation incrémentale dans ses réseaux de sous-traitance qui rayonnent quant à eux dans les segments connexes, notamment les services. Dans ce contexte, la politique structurelle du Land a consisté à réformer en profondeur son administration (compétences exclusives des Länder) : débureaucratisation, mise en réseau ('guichets uniques') des acteurs institutionnels auparavant très dispersés et cloisonnés, réforme des missions de l'administration publique et introduction du management qualité.

L'existence d'une longue réputation universitaire (le Land compte 9 universités ; celle de Fribourg fêtera ses 550 ans en 2007), de même que la multiplication des centres de recherche appliquée du Mittelstand, constituent un autre facteur de compétitivité potentielle dont le Land a su saisir l'enjeu stratégique en développant les passerelles entre la recherche et le marché, comme le rappelle une étude réalisée par la Fondation Bertelsmann. La réforme du financement des universités intervenue en 2002 en Allemagne (voir REA 66/04) a été bénéfique aux établissements du Bade-Wurtemberg. La part des financements publics étant désormais fixée au prorata des recettes propres de chacun d'entre eux, les universités du Bade-Wurtemberg battent un record depuis 2002 : la part des recettes propres par poste de professeur y est de $103000 €$, contre $80000 €$ seulement en moyenne allemande. L'attractivité du site explique la présence d'une population hautement qualifiée : près d'un dixième des citoyens du Land est diplômé de l'enseignement supérieur. Au plan de la formation initiale, le système scolaire du Land (les Länder sont souverains en la matière) est considéré comme l'un des plus performants d'Allemagne dans les études PISA de l'OCDE ou leurs équivalents allemands. Conjugué à la prédominance du Mittelstand où se recrute l'essentiel des entreprises dispensant une formation professionnelle (voir REA 69/04), cet état de fait explique que le taux de chômage du Land est le plus faible d'Allemagne, quelle que soit la cohorte considérée.

\section{Le dispositif de transfert et de recherche appliquée du Bade-Wurtemberg}

- 7 centres de recherche indépendants placés auprès des universités du Land. Ils ont été créés au cours des années 1980 Leur vocation est d'assurer le transfert des savoirs entre la recherche universitaire et l'industrie, via projets ou réseaux de coopération. Leurs directeurs sont employés de l'université auprès de laquelle est placé leur centre. Ces centres sont détenus par des fondations et soutenus par plus de 200 entreprises. Budget 2003 : plus de 49 millions $€$ au total ; le financement privé (entreprises) s'est élevé à 16 millions $€$. II s'agit du Forschungszentrum Informatik (Karlsruhe), du Naturwissenschaftliches und Medizinisches Institut (Tübingen), du Forschungsinstitut für angewandte Wissensverarbeitung (UIm), de I'Institut für Lasertechnologien in der Medizin und Messtechnik (UIm), de I'Institut für Mikroelektronik (Stuttgart), du Zentrum Fertigungstechnik (Stuttgart) et du Zentrum für Sonnenenergie- und Wasserstoff-Forschung (Stuttgart et UIm).

- 8 instituts de recherche professionnelle de branche. Ils ont été fondés par les fédérations industrielles et/ou sont soutenus par des associations de fondations avec pour mission d'accompagner les PME dans leur modernisation technique. Ceux du Land sont spécialisées dans les domaines suivants : textile (notamment technologies de production et chimie), chimie des métaux, pigments, microtechnologies, mécanique de précision/horlogerie et tannerie. S'y ajoute à Stuttgart la succursale du Süddeutsches Kunststoffzentrum Würzburg (SKZ, matières plastiques). Budget 2003 : 31 millions $€$; 10 millions de financement privé (projets).

- 14 Instituts Fraunhofer (sur les 58 d'outre-Rhin). Ces instituts réputés de recherche appliquée sont financés conjointement par le Bund $(90 \%)$ et le Land où ils sont implantés (10\%). Dans le Bade-Wurtemberg, leur budget total s'élevait à 227 millions $€$ en 2003 (recettes propres : 72 millions $€$ ). Leurs spécialisations : technologies de la construction (IBP, Stuttgart ; IRB, Stuttgart), de l'énergie (ISE, Fribourg), TIC (IITB, Karlsruhe), robotique (IPA, Stuttgart), mesures et technologie des capteurs (EMI, Fribourg ; IPM, Fribourg), ingénierie et process (IAO, Stuttgart ; ICT, Pfinztal ; IGB, Stuttgart ; ISI, Karlsruhe ; TEG, Stuttgart), matériaux (ISC, Wertheim ; IWM, Fribourg)

- 2 centres du réseau Helmoltz spécialisé dans la recherche fondamentale et le transfert. II pilote les grands programmes fédéraux d'innovation (technologies-clefs, énergie, sciences de la terre et de l'environnement, santé et structure de la matière ; voir REA 70/05). Leur financement est lui aussi conjoint ; le Land y contribue à hauteur de $10 \%$. Budget total 2003 : 350 millions $€$ (dont 19 millions e de ressources propres). Les deux centres installés dans le Bade-Wurtemberg sont : le Deutsches Zentrum für Luft- und Raumfahrt (DLR) avec deux sites, à Stuttgart (matériaux, énergie) et à Lampoldshausen (propulsion) ; le Forschungszentrum Kars/ruhe in der Helmholtz-Gemeinschaft (environnement, énergie, technologies des microsystèmes).

- La Fondation Steinbeis (Stuttgart) et son réseau de centres de transfert. Elle a été créée en 1971 avec pour mission d'accompagner le Mittelstand dans le processus de globalisation des activités et, ce faisant, de soutenir à son niveau la politique de compétitivité du Bade-Wurtemberg. Cette interaction s'est concrétisée en 1982 par la création d'un poste de Chargé du gouvernement pour le transfert des technologies; celui-ci assume en outre depuis 1983 les fonctions de président du directoire de la Fondation. Le réseau des 300 centres Steinbeis est réparti sur l'ensemble du territoire du Land, généralement à proximité des universités, où sont recrutés quelque 3030 enseignants et chercheurs (toutes disciplines). L'éventail des activités du réseau s'étend de la diffusion de l'information sur le développement des techniques et technologies ou l'élaboration d'études de marché au management et/ou pilotage de projet, sans oublier l'intelligence économique (voir dans ce numéro). Les recettes issues des activités sur contrat se sont élevées à 89 millions $€$ en 2003. 
La seule ressource naturelle notable du Bade-Wurtemberg étant son capital humain (et la légendaire inventivité de ses habitants), le gouvernement du Land a misé très tôt sur une politique de soutien aux approches innovantes des entreprises sur son territoire. Sa politique se contente de soutenir budgétairement les nombreuses structures de recherche appliquée et de transfert technologique ou de savoirs installées dans le Land. Elle décline certes à l'échelon régional les axes de la politique d'innovation et de recherche du Bund tout en l'insérant dans le champ européen. Mais elle suit plus encore ses orientations propres, déterminées avant tout par la structure économique bade-wurtembergeoise et les impératifs d'innovation auxquelles sont soumises ses branches dans la compétition mondiale. Dans ce contexte, le ministre de l'Economie du Land définit ainsi sa mission prioritaire: "soutenir les efforts conjoints entrepris par le monde de l'entreprise et le monde de la recherche pour développer des solutions innovantes orientées sur l'entreprise ". Sa politique se résume dès lors à créer les conditions visant le bon fonctionnement d'un système intégré d'interaction entre l'offre en matière de recherche et de technologies et la circulation des savoirs entre tous les acteurs (y compris publics) grâce au transfert. C'est ainsi qu'il soutient la compétitivité du site de production : en contribuant au financement $(77$ millions $€$ en 2003) de tous ces établissements de recherche qui jouent le rôle de passerelle entre la recherche fondamentale (universités, Instituts Max-Planck) et le marché, et qui figurent, avec les fédérations professionnelles et les partenaires sociaux, parmi ses partenaires privilégiés. Le dispositif est dense : il comprend notamment 7 centres de recherche indépendants placés auprès des universités, 8 instituts de recherche professionnelle de branche (sur les 54 recensés en Allemagne), 2 centres Helmholtz de recherche appliquée (sur 15), 14 instituts Fraunhofer (soit le quart du dispositif allemand). Sans oublier la Fondation Steinbeis, créée à Stuttgart en 1971, et à laquelle est dévolu un rôle d'interface entre la politique globale de compétitivité du Land et la modernisation des entreprises du Mittelstand.

La politique structurelle du Bade-Wurtemberg, établie dans la continuité, se limite au fond à aider les acteurs économiques du Land à développer leur potentiel propre en se fondant sur un système comme une culture d'activités et d'innovation en réseau. Dans cette approche, qui inclut le développement du public private partnership, le gouvernement se situe lui-même dans une logique de partenariat avec l'ensemble des acteurs économiques comme de la société civile et, cela va sans dire, avec le réseau des $12 \mathrm{CCl}$. Son rôle est dès lors de mettre à leur disposition les moyens leur permettant d'optimiser leur propre dynamique de croissance. Ces moyens sont certes budgétaires (118 millions $€$ pour le soutien au Mittelstand en 2004), et ils se cumulent avec les prêts consentis par la L-Bank au titre du soutien à la création ou la succession d'entreprises (1,5 milliard $€$ ). Ils concernent aussi l'aménagement du territoire, comme la construction d'un nouveau site pour la foire de Stuttgart, qui ouvrira ses portes en 2007, ou le développement des infrastructures de transport (conjointement avec le Bund, compétent pour les grands axes nationaux), vital pour la compétitivité d'une économie aussi ouverte. Le Land coopère avec la Suisse et l'Autriche pour le développement du réseau de la région du Lac de Constance, et attend avec impatience l'achèvement de la liaison TGV Paris-Stuttgart via Karlsruhe (et une hausse afférente de la contribution fédérale à son financement). Mais les véritables moyens de cette politique au long cours sont autres.

L'INNOVATION « NAIT DANS UN PROCESSUS où l'économie, la science, la formation et la société agissent en réseau, et dont le centre est l'individu en tant que porteur des savoirs ". Voici comment le ministère de l'Economie du Land décrit sa nouvelle politique de compétitivité globale dans la société du savoir. Cela passe par le soutien ciblé à certains clusters. Mais il ne faut pas se méprendre sur la nature de ce soutien. "La promotion de clusters n'a rien à voir avec une politique de soutien sectoriel; il ne s'agit pas de politique industrielle sous un autre nom » (van der Linde, 2005). II s'agit d'une approche globale dont on
... et soutien aux centres et réseaux de transfert
Une action placée au service des acteurs économiques 
trouve le reflet dans les quatre thématiques développées lors du dernier congrès des $\mathrm{CCl}$ du Land (24-11-05) : mobilité et infrastructures, formation/qualification, démographie, innovation/technologies. L'industrie du Bade-Wurtemberg n'a plus à faire la preuve de sa compétitivité. Mais les services doivent désormais prendre le relais. Quelle meilleure occasion pour en diffuser la prise de conscience que le Mondial de football dont plusieurs matches auront lieu dans le Land? Diverses institutions, dont les $\mathrm{CCl}$, ont lancé à l'automne un « concours du champion du monde des services Mondial 2006 " s'adressant à toutes les entreprises et associations. Mais l'enjeu crucial pour l'avenir du site est le développement de l'exportation des services, souvent en accompagnement de la vente de produits industriels : prestations techniques, d'ingénierie, mais aussi de santé ou de loisirs. Le Land et le réseau des $\mathrm{CCl}$ ont donc lancé en septembre 2005 une "Initiative pour l'exportation de services", afin de permettre aux entreprises du secteur de mieux croître à l'international et de consolider ainsi en retour l'emploi régional.

Ce nouvel axe s'insère dans une politique de compétitivité globale où le gouvernement du Land se contente généralement d'agir sur le cadre réservé aux activités et de jouer un rôle d'animateur au sein d'un ensemble de réseaux et partenariats qui se sont développés en grande partie spontanément au fil des décennies, mûs par les impératifs de compétitivité des entreprises. Voilà le véritable facteur structurel qui confère au Bade-Wurtemberg cette intelligence économique sur laquelle il a construit sa compétitivité et compte bien la développer à l'avenir. II s'agit là d'une culture, bien plus que de dispositifs institutionnels ou de moyens financiers (abondants et généreux au demeurant). Or cette culture, qui implique un benchmarking permanent, permet aussi de déceler les handicaps larvés et donc de développer à un stade précoce les outils permettant d'y remédier. C'est ainsi que la promotion du knowledge management fait partie des axes déclarés de la politique du Land, dans l'objectif d'une large diffusion, a fortiori dans le tissu des PME, des compétences-clefs de l'économie du savoir.

\section{Indications bibliographiques}

ARNDT O. ET AL., « Baden-Württemberg - Erfolg durch Wettbewerbsfähigkeit », étude réalisée par Prognos AG pour le compte de L-Bank, 2005

Baden-Württemberg. Eine kleine politische Landeskunde, Landeszentrale für politische Bildung Baden-Württemberg, 2003 (www.lpb.bwue.de)

" Baden-Württemberg », Länderanalysen, 2005 (www.bertelsmann-stiftung.de)

Bundesländer-Ranking, étude réalisée par IW-Consult $\mathrm{GmbH}$ et GWS GmbH en coopération avec l'Initiative Neue Soziale Marktwirtschaft et l'hebdomadaire Wirtschaftswoche, octobre 2005 (www.iwconsult.de)

BOURGEOIS I., "Le 'miracle' biotechnologique allemand», Regards sur l'économie allemande $\mathrm{n}^{\circ} 57 / 02$

GABEL M., "Chine, Inde, Brésil : les choix des entreprises allemandes », Regards sur l'économie allemande $n^{\circ} 70 / 05$, "Financement et vieillissement: le Mittelstand en mutation », Regards sur l'économie allemande $n^{\circ}$ 69/04 et « Commerce extérieur allemand : faiblesses et atouts ", Regards sur l'économie allemande $n^{\circ} 63 / 03$

LICHTBLAU K et al., "Erfolgsfaktoren von M+E-Clustern in Deutschland », IW-Trends, 2/05

Mittelstandsbericht 2005, Wirtschaftsministerium Baden-Wurttemberg, (www.wm.badenwuerttemberg.de)

DE LA PAIX R., "Les politiques d'innovation en Alsace et dans le Pays de Bade », Regards sur l'économie allemande $n^{\circ} 54 / 01$ et « Capital-risque pour les jeunes entreprises du BadeWurtemberg : le rôle des pouvoirs publics ", Regards sur l'économie allemande n 44/99

VAN DER LINDE C., "Cluster und regionale Wettbewerbsfähigkeit. Wie Cluster entstehen, wirken und aufgewertet werden ", in Cernavin 0. et al. (eds), Cluster und Wettbewerbsfähigkeit von Regionen. Erfolgsfaktoren regionaler Wirtschaftsentwicklung, Berlin, 2005

WeInmann TH., "Baden-Württemberg ist die innovativste Region der EU », Statistisches Monatsheft Baden-Württemberg, 10/04

WERNER J., FISCHER B., " Europäische Metropolregionen im Vergleich », Statistisches Monatsheft Baden-Württemberg 7/2005

Wirtschaftsnahe Forschungseinrichtungen in Baden-Wurttemberg, Wirtschaftsministerium Baden-Wurttemberg, 2004 (www.wm.baden-wuerttemberg.de)

www : baden-wuerttemberg.de ; bw-ihk.de ; bw-i.de ; fraunhofer.de ; steinbeis.de

Zukunftsatlas 2004, Prognos AG (www.prognos.com/zukunftsatlas) 